\title{
Patterns of Normal Human Brain Plasticity After Practice and Their Implications for Neurorehabilitation
}

\author{
Clare Kelly, PhD, John J. Foxe, PhD, Hugh Garavan, PhD
}

ABSTRACT. Kelly C, Foxe JJ, Garavan H. Patterns of normal human brain plasticity after practice and their implications for neurorehabilitation. Arch Phys Med Rehabil 2006;87(12 Suppl 2):S20-9.

Objectives: To illustrate how our knowledge about normal patterns of experience-induced plasticity can provide insights into the mechanisms of neurorehabilitation; to provide an overview of the practice-effects literature in order to simplify and amalgamate a large number of heterogeneous findings and identify typical patterns of practice effects and their determining factors; and to concentrate on the impact of practice on higher cognitive functions, such as working memory, and present some preliminary but promising behavioral data that show how practice on a complex cognitive task can benefit cognitive functioning more generally.

Data Sources: We performed a systematic search for peerreviewed journal articles using computerized databases (PubMed, ISI Web of Science, PsycINFO).

Data Selection: Neuroimaging studies using functional magnetic resonance imaging (fMRI) or positron-emission tomography (PET) to examine functional activation changes as a result of practice on sensory, motor, or cognitive tasks in normal (healthy) populations were included in the review. Further studies were identified that examined the effects of rehabilitative training on functional activations in clinical populations using fMRI or PET.

Data Extraction: Important characteristics of the selected studies were summarized in a systematic manner so to enable the extraction of specific factors impacting on the pattern of practice effects observed.

Data Synthesis: We identified a number of factors that impact on the patterns of practice effects observed and discuss how the insights gained from the study of healthy populations can by applied to rehabilitation of cognitive deficits in clinical populations.

Conclusions: Progress in our understanding of neurorehabilitative plasticity will be enabled by neuroimaging examinations of cognitive rehabilitation training grounded in a knowl-

From the School of Psychology and Trinity College Institute of Neuroscience, Trinity College, Dublin, Ireland (Kelly, Garavan); The Cognitive Neurophysiology Laboratory, Nathan S. Kline Institute for Psychiatric Research, Program in Cognitive Neuroscience and Schizophrenia, Orangeburg, NY (Foxe, Garavan); Program in Cognitive Neuroscience, Department of Psychology, City College of the City University of New York, New York, NY (Foxe); and Department of Psychiatry and Behavioral Medicine, Medical College of Wisconsin, Milwaukee, WI (Garavan).

Supported by Trinity College Institute of Neuroscience (National Education Authority's Programme for Research in Third Level Institutions grant), U.S. Public Health Service (grant no. DA14100), General Clinical Research Centers (grant no. M01 RR00058), and National Institute of Mental Health (grant nos. MH63434, MH65350).

No commercial party having a direct financial interest in the results of the research supporting this article has or will confer a benefit upon the author(s) or upon any organization with which the author(s) is/are associated.

Reprint requests to Hugh Garavan, PhD, School of Psychology, Trinity College

Dublin, Dublin 2, Ireland, e-mail: Hugh.Garavan@tcd.ie.

0003-9993/06/8712S-10975\$32.00/0

doi:10.1016/j.apmr.2006.08.333 edge of normal (healthy) patterns of brain activation and practice-induced plasticity.

Key Words: Neuronal plasticity; Rehabilitation.

(C) 2006 by the American Congress of Rehabilitation Medicine

$\mathbf{T}$ HE DISCOVERY THAT experience-driven changes in the human brain can occur from a synaptic to a cortical level throughout the life span has been termed a nascent revolution in cognitive neuroscience, ${ }^{1}$ overthrowing the long-held belief that the adult brain is hard-wired and resistant to change. ${ }^{2}$ Research with both animal models and humans has shown that the organization of the adult cerebral cortex can change substantially as a result of practice and experience. ${ }^{3,4}$ Furthermore, experience-dependent change can occur at multiple levels of the central nervous system, from the molecular or synaptic level to the level of cortical maps and large-scale neural networks. $^{2}$ These discoveries challenge us to investigate how it is that the brain changes in response to experience. Moreover, if the neuroanatomic, neurochemical, and functional changes that take place in response to practice also underlie the recovery of function following damage to the brain, then this knowledge will aid in our understanding of the mechanisms of repair and recovery in damaged or malfunctioning brains. Neuroimaging methods such as positron-emission tomography (PET) and functional magnetic resonance imaging (fMRI) are excellent tools in this endeavor, enabling the examination of how the brain changes in response to practice or training on a particular task. The aim of this review was to illustrate how our knowledge about normal patterns of experience-induced plasticity can provide insights into the mechanisms of neurorehabilitation and to suggest how best to facilitate this rehabilitation through cognitive and behavioral interventions.

There is ample evidence to suggest that, after injury, plastic changes occur in the brain in order to compensate for loss of function in damaged areas. ${ }^{5-7}$ These changes will not always be beneficial, however, nor is substantial recovery or restitution of function likely in the absence of targeted intervention. ${ }^{6}$ What pattern of practice-induced functional change is likely to occur in response to such interventions? Although the answer to this question is likely to depend principally on the extent and severity of the injury, there are a number of insights to be gained from the examination of normal practice-induced plasticity that may apply to rehabilitative situations. In the first part of this review, we provide a brief overview of the patterns of normal brain plasticity that occur in response to practice, with a particular emphasis on the potential for practice of cognitive functions. To demonstrate this potential, we provide some preliminary but promising behavioral data that show how practice on higher cognitive tasks can benefit cognitive functioning more generally. In the second section of the review, we discuss the small number of studies that have used neuroimaging methods to examine the neural consequences of cognitive training in clinical populations. These studies provide an illustration of how the insights gained from the examination of neuroplasticity in normative populations, discussed in the first 

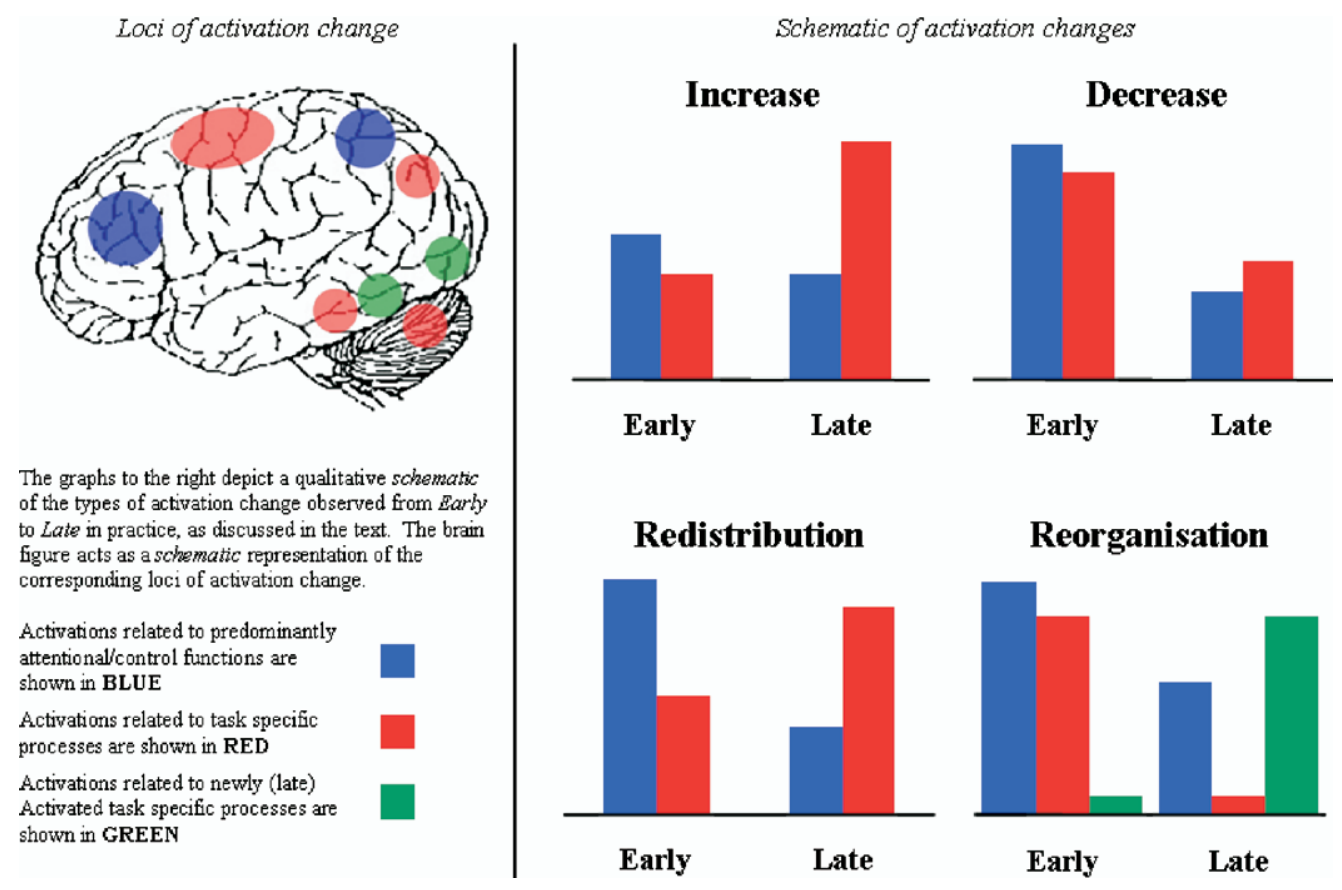

Fig 1. Schematic of practice-related changes in functional activations. The figure represents a highly schematized depiction of the changes in levels of activation and some of the potential functional loci of those changes associated with the different types of practice-related changes in functional activation described in the text. Adapted with permission from Kelly and Garavan. ${ }^{8}$

half of the review, can by applied to rehabilitation of cognitive deficits.

\section{PATTERNS OF PRACTICE-RELATED ACTIVATION CHANGE}

The literature dealing with the effect of practice on the functional anatomy of task performance is extensive and complex, comprising a wide range of studies from disparate research perspectives. Researchers have used a variety of tasks and paradigms and a diversity of practice techniques and schedules; consequently, there is a corresponding diversity in the pattern of results reported. Different studies report increased or expanded activations, decreased activations, or a reorganization of the functional activations underlying task performance. Briefly, we will provide an organizing framework that will guide understanding and interpretation of these results. For a more extensive review of the practice effects literature, we refer readers to Kelly and Garavan. ${ }^{8}$

Human functional neuroimaging studies investigate the changes in brain activation that occur as a result of practice on a range of motor, visuomotor, perceptual, and cognitive tasks. In this section, we distinguish 3 main patterns of practicerelated activation change and provide illustrative examples from each of these different task domains. Figure 1 provides a schematic view of each of the patterns of activation change.

\section{Patterns of Practice-Related Activation Change}

Activation decreases. Decreases in the extent or intensity of activations are observed in the majority of studies examining task practice (albeit often in combination with other changes; see Reorganization of Activation: Functional Reorganization of Activations, below). The primary mechanism proposed to underlie activation decreases is increased neural efficiency, which is understood to reflect a sharpening of the response in a particular neural network so that fewer neurons now fire strongly in response to a particular task or stimulus. ${ }^{9}$ Decreases in activation therefore represent a more robust and efficient neural representation ${ }^{10}$ or a more precise functional circuit. ${ }^{11}$

A pattern of decreased activation is typically seen after practice on higher cognitive tasks, such as working memory tasks. For example, Garavan et al ${ }^{11}$ examined the effect of moderate practice on a visuospatial working-memory task. In this task, participants were required to hold in mind the location (on a computer screen) of a target circle and, after a delay of 3 seconds, to indicate whether the location of a probe circle matched that of the previously presented target circle. Garavan observed activation decreases in the majority of areas activated during task performance (prefrontal, parietal, occipital cortex) as a result of relatively brief (20-min) practice. This finding was understood to reflect an increase in neural efficiency. This increase in neural efficiency was hypothesized to underlie improvements in task performance that were observed over the practice period.

Although there are relatively few studies that examine changes in functional activation as a result of practice on cognitive tasks, such studies have typically observed a pattern of activation decreases. Decreased functional activations were observed by Jansma et $\mathrm{al}^{12}$ after practice on a verbal Sternberg task, Sayala et al ${ }^{13}$ after practice on an object/spatial working memory task, and Hempel et $\mathrm{al}^{14}$ after practice on an $n$-back task. Decreases in functional activations have also been observed after practice on other types of higher cognitive task, such as the Tower of London problem ${ }^{15}$ and tests of verbal free recall. $^{16,17}$ For example, Haier et al ${ }^{18}$ used PET to examine changes in glucose metabolic rate across cortical areas activated during performance of the computer game Tetris, a complex visuospatial-motor task. Haier observed a widespread decrease in glucose metabolic rate after several weeks of moderate training. These decreases in glucose metabolic rate correlated with improvements in task performance. Importantly, 
this correlation suggests that the brains of subjects doing the task well function more efficiently than the brains of poorer performers, consistent with the hypothesis that functional decreases in activation reflect more efficient information processing in the brain.

Activation increases. The term increases in activation refers to both practice-related expansions in the spatial extent of cortical representations and increases in the strength or amplitude of activations. The lack of distinction between these 2 is likely based on the fact that expanded representations may be undetectable at the spatial resolution of most neuroimaging studies or may be masked by spatial smoothing analytic methods. On a neural level, increases in activation may reflect recruitment of additional cortical units with practice, which is seen in topographically organized cortex as an increase in the spatial extent of activation. Alternatively, increases may reflect a strengthening of the response within a particular region, observed as an increase in the level of activity within that region. ${ }^{9}$

An increase in the extent or amplitude of functional activations is most typically observed after extensive practice on sensory or motor tasks, in contrast to decreases in activation typically observed after practice on cognitive tasks. For example, the long-term motor training that is associated with extensive practice on a musical instrument can induce an expansion of digit representations in somatosensory cortex. ${ }^{19-2}$ In an empirical investigation of this effect, Karni et $\mathrm{al}^{22}$ used fMRI to examine functional activations in primary motor cortex (M1) while participants performed a finger-to-thumb opposition sequence that had been practiced for 10 to 20 minutes each day over several weeks. Performance of the trained sequence reached asymptote after 3 weeks, from which time it evoked an activation map that was consistently larger than that of a control sequence that was performed only during fMRI scanning. The larger activation map reflected an expansion of the representation of the trained sequence in $\mathrm{M} 1$ as a result of the recruitment of additional M1 units. ${ }^{22}$ One caveat concerning the Karni study is that they only examined activation changes in M1 - the acquisition volume did not include other areas of the brain. As a consequence, the authors were unable to observe other practice-related changes in activation that may have been present. Consistent with this suggestion is the fact that many studies that have observed activation increases as a result of practice on sensory or motor tasks also observe concomitant activation decreases in other regions of the brain and are better described by the pattern of redistribution, discussed in the next section.

Reorganization of activation. A reorganization of functional activations as a result of practice is the most commonly observed pattern. We distinguish 2 types of practice-related reorganization, examples of which are discussed below.

Reorganization of activation: redistribution of functional activations. This pattern of practice-related change reflects a quantitative change in the pattern of activations underlying task performance. It constitutes a combination of increases and decreases in functional activations such that the task activation map generally contains the same areas at the end as at the beginning of practice, but the levels of activation within those areas have changed. In addition, the cognitive processes engaged by the task remain largely unchanged by practice.

Reorganization of activation: functional reorganization of activations. This pattern of activation change is the result of a qualitative shift in the cognitive processes underlying task performance and a change in the locus of activations as a result of practice. This shift, known as "process switching"9 means that neurobiologically and cognitively, different tasks are being performed at the beginning and end of practice, and this results in a coordinated increase and decrease of activation in separate brain regions. Reduced activity in a particular region reflects less engagement of a particular cognitive process, whereas increased activation in a different region reflects the engagement of an alternative cognitive system or the development of new representations or processes. ${ }^{9}$

It is important to note that, in the context of the practice effects literature, which has proceeded under the umbrella of cognitive neuroscience, the term reorganization has carried a different meaning than in the context of the clinical literature dealing with recovery of function after damage or injury to the brain. In the latter context, reorganization has typically been used to describe a pattern of activation in which parts of the brain unaffected by the injury become engaged by a particular task or cognitive function after injury. That is, it refers to a pattern of compensatory activation in which different brain areas are recruited to perform a function or task that usually engaged the injured region. Such compensatory activations may later diminish in association with recovery and reactivation of the damaged region ("normalization"). We will return to a discussion of this pattern in the second section of this review, when we discuss functional imaging studies of rehabilitation and recovery of function after brain injury. In contrast, we consider a functional reorganization of activations to reflect a qualitative change in the cognitive processes engaged by the task as a result of practice, which results in the engagement of different brain regions. We will illustrate this pattern with examples below.

\section{Redistribution}

Redistribution constitutes a combination of increases and decreases in functional activations such that the task activation map generally contains the same areas at the end as at the beginning of practice, but the levels of activation within those areas have changed. The functional anatomy of the task therefore remains basically the same, but the contribution of specific areas to task performance changes as a result of practice. This pattern most typically reflects a decreased demand on control or attentional processes (wherein activation decreases with practice) and an increased demand on storage and processing in task-specific areas (wherein activation increases) and has been discussed previously by Petersen et $\mathrm{al}^{23}$ in terms of a "scaffolding-storage" "framework. According to this framework a scaffolding set of regions is used to cope with novel demands during unskilled, effortful performance. After practice, processes or associations are more efficiently stored and accessed and the scaffolding network falls away, evinced by decreased activation in those scaffolding attentional and control areas (eg, prefrontal cortex [PFC], anterior cingulate cortex [ACC], posterior parietal cortex). A coordinated increase in activation is observed in those areas underlying task-specific processes (ie, in representational cortex - primary and secondary sensory or motor cortex, or areas related to the storage of representations such as parietal or temporal cortex).

A study by Shadmehr and Holcomb ${ }^{24}$ illustrates this pattern of activation change. In their study, participants used a robotic arm to reach for a target but had to learn to adapt their reaching movements in response to a distortion imposed by the robotic arm. With practice, their performance became highly skilled and converged on that of a condition in which participants experienced no distortion and were able to move normally. Shadmehr and Holcomb used PET to examine brain activations during a recall session that took place 5.5 hours after the last practice session. A redistribution of activations from frontal to posterior (parietal cortex, cerebellum) areas was observed. 
Interestingly, these changes occurred in the absence of any further performance changes, emphasizing the complexity of the brain's response to repeated task experience. Shadmehr and Holcomb proposed that the acquisition of skilled movement is mediated through PFC structures. Then, with time and practice, as automation occurs, motor structures such as the cerebellum assume a greater role in task performance and possibly become the site of motor memory.

A similar pattern of redistribution of activations was also observed in studies involving visuomotor tasks ${ }^{25-28}$ and motor tasks $^{29,30}$ and has also been shown by studies that show that explicit attention to task performance is associated with increased activations in prefrontal and parietal control areas, whereas implicit or procedural task performance is associated with increased activations in task-specific motor areas. ${ }^{31-33}$

The key to redistribution of functional activations is the practice-related decrease in activation in areas underlying cognitive and attentional control, whereas activations in taskspecific tend to increase. It is likely that practice-related decreases in the level of activation in attentional and controlrelated areas of the brain are common to almost all practiceeffects studies. That is, during naive performance of most tasks, there will be some degree of supporting activation in attentional and control areas that drops off as one becomes more practiced on the task. In fact, this pattern may be prevalent in rehabilitative training situations, as part of the compensatory response of the damaged brain. That is, attentional and control processes may be recruited during the initial stages of recovery in order to "scaffold" impaired functioning. As recovery proceeds, these compensatory recruitments may diminish. This particular pattern of activation change has been observed in studies involving clinical populations, ${ }^{34-36}$ a discussion of which is provided in the final section of the review.

\section{Functional Reorganization of Activations: "Process Switching"}

As mentioned, redistribution reflects a quantitative change in the magnitude of functional activations as a result of practice. Such a quantitative change occurs when the cognitive processes involved in task performance are not substantially altered by practice, even after extensive practice or changes in behavioral output. In contrast, a functional reorganization of activations represents a qualitative shift in the cognitive processes underlying task performance and a change in the locus of activations as a result of practice: "process switching." 9 Thus, the determination of whether the cognitive processes in operation early in practice persist until late in practice, or whether a process switch has occurred, is a critical factor in distinguishing redistribution (persistence) from reorganization (switch).

A study by Raichle et $\mathrm{al}^{37}$ provides the clearest example of how a qualitative shift in task processes produces a functional reorganization of activations. Using PET, they imaged brain activity while participants generated verbs to a list of nouns (eg, when presented with the noun cake, one might respond with the verb eat). The generate task was repeated for several blocks $(\approx 15 \mathrm{~min}$ of practice); then participants were scanned again while they generated verbs to both the original list and a new list of nouns. Practice significantly reduced reaction time and led to the development of stereotyped responses to approximately $90 \%$ of the nouns. Raichle ${ }^{37}$ observed a reorganization of functional anatomy underlying the task-reduced activation in ACC, left PFC, and right cerebellum and increased activity in insular cortex. It is clear that this reorganization was a result of the practice-related change in the cognitive processes involved in performing the verb-generation task-there was a switch away from effortful, semantic processing and selection from an unlimited set of responses early in performance, to paired-associate episodic recall (stereotyped responses to each noun), minimizing effort and semantic processing. In support of this interpretation, when activations during verb generation to the new word list were examined, the researchers observed reactivation of the frontal areas and decreased activation of left insular cortex. A reorganization of functional activations has also been observed in other studies that show a similar practicerelated shift in the cognitive processes involved in task performance, including mirror reading tasks, ${ }^{38,39}$ artificial grammar learning, ${ }^{40}$ and free-recall tasks. ${ }^{41}$

Quantitative change, task domain, and mechanisms of plasticity. On the basis of the preceding description of the primary patterns of practice-related activation change, we can see that there is a distinction between quantitative and qualitative practice-related changes in functional activations. The reader may also have noticed that we may further differentiate patterns of quantitative change in activation (activation increases and activation decreases) on the basis of the task domain. That is, activation decreases are most typically observed after practice on complex cognitive tasks (eg, those involving working memory), whereas activation increases are typically observed in sensory or motor tasks, which place less demand on cognitive processes, particularly after practice (ie, the kinds of tasks that the performance of which one might think of as a "skill"). This dissociation is likely to reflect divergent plastic responses associated with the different task domains. That is, mechanisms of plasticity in association or nonprimary cortex, which typically subserve higher cognitive processes, may differ from those in topographically organized motor and sensory cortex.

Illustrative of the dissociation are studies that show expanded representations in topographic sensory and motor cortex as a result of extensive experience with a particular stimulus type. For example, expanded representations have been observed in somatosensory cortex for the dominant index finger of Braille readers compared with that of non-Braille readers ${ }^{42}$ for left-hand digits in string players compared with controls ${ }^{19}$; and in the primary auditory cortex for piano tones in musicians relative to nonmusicians. ${ }^{43}$ The primary mechanism of this expansion is thought to be modifications occurring in the horizontal connectivity of primary cortex. ${ }^{2}$ Modifications in horizontal connectivity in primary cortex are proposed to result from changes in synaptic efficacy, possibly through long-term potentiation-like mechanisms and are also suggested to underlie experience-related plasticity in other nonmotor cortex, for example, visual cortex. ${ }^{20}$ On a cognitive level, these practice-related changes in representations have been associated with the development of a specific memory trace-an "internal model" 24 of the practiced task. Thus, in sensory and motor tasks, which involve topographic cortical representations, a primary outcome of practice takes the form of an expanded representation within primary cortex as a result of increased connectivity within that area.

In contrast, the representational demands of higher-level cognitive tasks are such that a more distributed network of brain regions is observed to underlie task performance. The specific operations and stimuli involved in the performance of cognitive tasks may not therefore lend themselves to the formation of the same kind of long-term localized and specific memory trace that has been hypothesized to exist for sensory and motor tasks. Instead, practice on cognitive tasks is hypothesized to increase the efficiency of the distributed task network, reflected in a decrease in activation in the network of brain areas underlying task performance, as studies ${ }^{11,12,15,44,45}$ exam- 
ining the effect of practice on higher cognitive (eg, working memory) tasks have shown. This pattern is consistent with electrophysiologic research with monkeys, which shows weaker neuronal activity in response to practiced or familiar stimuli or task conditions. ${ }^{46-49}$ By analogy, although practicerelated changes in activation on sensorimotor tasks may predominantly take the form of increased connectivity within primary cortex, the type of plasticity and activation change associated with practice on higher cognitive tasks is the result of changes in connectivity between nodes of a highly distributed network of functional areas. This suggestion is supported by studies showing changes in connectivity between regions as activity within those regions decreased as a result of learning. ${ }^{50,51}$

A consequence of these differential mechanisms of plasticity resulting from practice on higher cognitive tasks, compared with sensory and motor tasks, is that extensive practice on the latter type of tasks typically produces highly specific improvement in the trained task only. That is, practice-related improvements in performance do not generalize to different task sequences or stimuli. ${ }^{52,53}$ Although few studies ${ }^{54-56}$ have examined the transfer of practice-related improvement in higher-level cognitive tasks, the results of those studies are promising, and indicate that significant transfer of practicerelated improvement to similar cognitive tasks can occur. This finding is very interesting when considered in terms of its potential application to rehabilitative situations. We will return to a discussion of rehabilitative applications in the second section of this review.

Individual differences. One important factor in determining the magnitude of activations observed in response to a cognitive task is the existence of basal interindividual differences in performance. Many of these differences are thought to be a phenotypic expression of variations in dopamine-related genes that affect people's working memory capacity and the ability to exert control over attention. 57 In support of this, functional imaging studies have shown that there is a relation between polymorphisms in the catechol- $O$-methyltransferase (COMT) gene, which affect levels of dopamine in prefrontal systems, and brain activity in PFC. During working memory tasks, participants with a polymorphism related to high levels of dopamine (Met allele) exhibit less task-related brain activity than subjects with the polymorphism related to low dopamine activity (Val allele). Met allele participants also perform significantly better on tasks of cognitive flexibility and working memory than the Val allele group. ${ }^{58-60}$ Thus, individual genetic variations in the COMT polymorphism affect basal levels of PFC dopamine. Baseline individual differences in levels of PFC dopamine, in turn, affect the efficiency of PFC functioning during working memory. Specifically, there appears to be an inverted U-shaped relation between dopamine levels and PFC function and activation. ${ }^{57}$

Such individual differences are likely to interact with the effects of practice. That is, in addition to genetic factors, a person's previous experience with the particular task or skill will also alter his/her position on the inverted U-shaped function that describes activation levels. A very different pattern of activation change may be observed in participants with a high level of familiarity compared with those naive with respect to the particular task or stimulus used. This effect was shown by Hund-Georgiadis and von Cramon, ${ }^{20}$ in a comparison of the functional networks activated by highly skilled musicians and nonmusicians during performance of a motor tapping task. Musicians activated a smaller neural network than nonmusicians. Furthermore, musicians showed a practice-related increase in M1, whereas nonmusicians did not. Such practice- related increases in M1 are typically observed after extensive (ie, several weeks) practice in nonmusicians. ${ }^{22}$ Thus, the pattern of practice-related activation change observed in musicians resembled the effects of much more extensive practice in nonmusicians. Pre-practice experience accelerated practice-related changes in functional activation in the brain. This finding may have a parallel in the observation that recovery from brain damage is greatest in those who were better educated or more intellectually active before the brain insult. ${ }^{61-63}$

Thus, the direction and magnitude of practice-related activation change may be a function of a person's pre-existing cognitive abilities and experience. This emphasizes the importance of taking into account both basal and practice-induced differences in performance and activation in the examination of practice effects. With regard to rehabilitation, such individual differences may complicate the expression of the effects of training. On the other hand, the existence of baseline individual differences in cognitive function related to underlying genotypic differences may help identify those people likely to benefit most from such practice. ${ }^{64,65}$

The role of other factors. Briefly, there are several other factors that can have a significant effect on the pattern of practice effects seen, which will be important to take into account in a rehabilitative context. These have been discussed in greater detail elsewhere. ${ }^{8}$

Changes in performance are often an inherent characteristic of practice. Such changes include direct behavioral effects such as increased accuracy, decreased reaction time, increased processing speed, and secondary task-related effects such as reduced errors, decreased time on task, and more frequent responses. A confound arises here because it is possible that any changes in activation observed are secondary to the behavioral change, rather than to the presumed underlying practice-driven changes in neural efficiency or cortical representations. 9,66 For example, van Mier et al $^{67}$ observed a practice-related increase in M1 activation that is confounded by a concurrent increase in the velocity of motor output, which may in itself explain the increased M1 activity. Neural activation also differs as a function of duty cycle (time on task) or task pacing, which can also change as a result of practice. The converse problem arises in those cases in which practice does not produce changes in performance. In the absence of behavioral change, how can changes in activation be interpreted? Several studies ${ }^{24,45,68}$ have observed meaningful practice-related changes in activations in the absence of concomitant behavioral changes. Although in such cases it is important to rule out fatigue or decreases in motivation as potential explanations for the activation changes observed, these observations provide remarkable demonstrations of how the brain produces overtly identical behavior despite often substantial changes in the underlying functional anatomy, an observation that has clear implications for research with brain-injured populations.

The point in practice at which participants are imaged will have a significant effect on the levels of activation observed. To make strong conclusions about the effects of practice in any study, researchers must be sure they have imaged the entire window of practice-related effects. A recent study by Hempel et $\mathrm{al}^{14}$ highlights this issue. They examined the effects of training on an $n$-back working memory task over a period of 4 weeks. They observed initial increases in activation in taskspecific areas followed by decreases in activation by the end of training and that decreases in activation values are associated with the consolidation of performance gains after extensive practice. They concluded that training-related changes in activation follow an inverted U-shaped quadratic function, with initial increases followed by later decreases. 
Finally, a potential confound also arises from those areas associated with task-irrelevant processing. These areas are proposed to be involved in "default" brain processing and typically become deactivated during task performance, relative to a rest state. ${ }^{69,70}$ Practice and increasing automaticity may lead to a decrease in the requirement for attentional suppression of irrelevant information processing, leading to a decrease in the level of deactivation in these task-irrelevant areas. Apparent increases in activation may therefore be spurious, a reflection of these reduced deactivations. This may be an important consideration in studies of clinical populations who show abnormalities in the levels of deactivation in the "default" resting network during the performance of cognitive tasks. ${ }^{71,72}$

The preceding brief review has summarized the predominant patterns of brain change associated with practice and the task domains and conditions in which these different patterns are observed and has identified a number of methodologic issues that bear on interpreting brain changes after task practice. We have noted that the majority of empirical studies focus on the training of sensory and motor functions rather than higher cognitive functions, an unfortunate imbalance given the importance and difficulty in rehabilitating these higher functions. However, emerging evidence suggests that complex cognitive functions can indeed be practiced. This prospect is of particular interest in the context of the centrality of cognitive control processes to cognition and behavior. Deficits in cognitive control have long been identified as central to a number of clinical conditions associated with damage or dysfunction in frontal cortex. For example, dysfunctional cognitive control is central to the neuropsychologic profile of Tourette's syndrome, ${ }^{73,74}$ obsessive-compulsive disorder, ${ }^{75,76}$ attention-deficit hyperactivity disorder (ADHD), ${ }^{77,78}$ schizophrenia, ${ }^{79,80}$ and traumatic brain injury. ${ }^{81,82}$ Cognitive control deficits also show significant declines with increasing age. ${ }^{83-85} \mathrm{~A}$ number of studies have already shown the efficacy of cognitive rehabilitation for such groups. ${ }^{86-88}$

The immense potential for practice on cognitive control processes is suggested by the theoretic framework in which individual differences in cognitive control (measured in terms of working memory capacity) are directly related to individual differences in performance across a wide range of tasks, with recourse to the concept of general fluid intelligence. ${ }^{89-91} \mathrm{Be}-$ cause working memory processes are thought to form the core of cognitive control functions, ${ }^{10,89-92}$ one might ask whether flexible cognitive control can be practiced by targeting working memory processes.

In a preliminary investigation of the potential for training cognitive control, we examined the behavioral effects of extensive practice on a challenging version of the $n$-back working memory task in a small group of healthy participants. In the $n$-back task, participants are presented with a sequence of stimuli (eg, letters) and are asked to judge whether the current stimulus matches the one preceding it by $n$ number of places, that is, the stimulus $n$-back. This task was previously identified as tapping those components of attentional control thought to underlie individual differences in general fluid intelligence and performance across diverse higher cognitive tasks. ${ }^{93}$ Specifically, Gray et $\mathrm{al}^{93}$ showed that high-interference "lure" trials place an increased demand on cognitive control, and performance and activation (measured by fMRI) during these trials correlated with participants' general fluid intelligence. We used a similar working memory task in our preliminary study, in which 6 participants took part in 18 training sessions. In each session the participants performed 384 trials of a verbal 4-back working memory task, which also included "lure" trials (3- and 5-back). Participants indicated whether each letter matched ("match" trials) or did not match ("nonmatch" trials) the letter seen 4 items previously (4-back). Their performance on the task was assessed in a pre- and post-training evaluation session. We also assessed generalization of practice-related improvement on 2 other demanding working memory tasks (a spatial $n$-back task, a verbal Sternberg task), on which participants received no practice.

Statistical analysis of performance as measured during the pre- and post-training evaluation sessions indicated a significant effect of practice on accuracy and reaction time for each of the trial types in the 4-back task (match, nonmatch, and lure trials, $P<.05)$. Importantly, although responses to lure stimuli were initially less accurate, slower than responses to nonlure and nonmatch trials, and slower than match trials, these differences were no longer significant after practice. This shows that lure trials initially elicited greater interference than the other trials, consistent with Gray. ${ }^{93}$ However, this interference was ameliorated with practice, and lure trials were no longer behaviorally dissociable from nonlure (noninterference) trials after practice. In addition, participants showed large and significant performance improvements on the "transfer" tasks, the spatial 4-back task, and the verbal Sternberg task between the pre- and post-training sessions, in the absence of intermediate practice on those tasks.

These data suggest that the cognitive mechanisms of attentional control are amenable to practice-related improvement. That is, that fundamental cognitive mechanisms thought to underlie performance across diverse cognitive tasks ${ }^{10,89-92}$ - and ultimately, adaptation to novel situations and successful integration within our complex world-can be improved through practice. The data suggest that complex cognitive abilities are not a fixed and immutable characteristic of a person, but instead, are amenable to change and improvement. An important implication is that deficits in cognitive performance occurring as a consequence of impairment in fundamental mechanisms of attentional or cognitive control may be ameliorated or rehabilitated through practice and training on tasks that specifically tap attentional control. Initial positive demonstrations of this application have been provided by Klingberg et $\mathrm{al}^{55,56}$ in children with ADHD. In those studies, working memory training increased working memory span, and this practice effect generalized to improved performance on untrained working memory tasks, inhibitory control, and reasoning (a measure of general fluid intelligence). Parent ratings indicated a significant reduction of inattention and hyperactivity symptoms, and the earlier ${ }^{55}$ study also showed a reduction in motor activity as a result of training. Several other studies ${ }^{86,87,94-96}$ have also shown positive effects of training on problem solving tasks (eg, the Tower of London problem), working memory, or attention training in clinical or patient groups.

Despite the importance of these behavioral findings, progress in our understanding of their application and utility to clinical populations has been limited by a lack of neuroimaging examinations of the effects of cognitive rehabilitation training. In the next section, we describe some of the emerging studies in this area and illustrate how they are informed by an understanding of normal brain plasticity.

\section{FUNCTIONAL NEUROIMAGING STUDIES OF REHABILITATIVE COGNITIVE TRAINING}

The preceding review of neuroimaging literature and the preliminary results our behavioral examination should be instructive for investigating neurorehabilitative plasticity. To date, however, neuroimaging of practice-related changes in performance and functional activations in patient groups has received little attention. Nonetheless, studies in this area are 
beginning to appear, and a very small number of studies ${ }^{34,35,97}$ have investigated the impact of cognitive training and performance improvement on functional activations in clinical groups. The results of these studies suggest that rehabilitative training of cognitive function can restore and even normalize activation. For example, functional neuroimaging studies ${ }^{98-100}$ have shown that dyslexic people show lower levels of activation in left temporoparietal cortex, which is associated with a deficit in phonologic processing. Temple et $\mathrm{al}^{35}$ imaged dyslexic children during a phonologic processing task before and after a remediation program that involved training on auditory processing and oral language skills. Training improved language processing and reading performance. Training also increased activity in several brain areas including temporoparietal cortex and the left inferior frontal gyrus, bringing brain activations closer to those of healthy children - a normalization of functional activations. In addition, the dyslexic participants showed compensatory activations in right-hemisphere areas analogous to left-hemisphere language processing areas in persons with normal reading ability, and in attention control areas such as the ACC. The authors suggested that many of these compensatory activations would diminish with further training and improvements to reading performance and that this would be accompanied by further normalization of activation in temporoparietal and frontal areas. These expectations are consistent with the pattern of redistribution of activations, described in section 1, which predicts that reliance on additional attentional and control circuits will diminish with practice.

A similar normalization of brain activations was observed by Wexler et $\mathrm{al}^{97}$ who examined the effect of training-related improvements in verbal working memory in schizophrenic patients. The patients trained on an auditory verbal working memory task 4 to 5 times a week for 10 weeks. During training, task difficulty automatically increased on the basis of successful performance. Improvements in performance on the verbal working memory task were associated with increased activation in left inferior frontal cortex, an area that initially showed hypoactivity. In 1 patient who continued training for a further 5 weeks, further performance improvements and normalization of brain activation were observed.

Working with cases of brain damage rather than dysfunction, Sturm et $\mathrm{al}^{34}$ examined training-induced changes in functional activation in patients with right-hemisphere vascular damage. These stroke patients initially displayed impaired attentional functioning. They performed a computerized alertness training program for 4 weeks. Functional activations during performance of another (unpracticed) alertness task were assessed pre- and post-training with PET. In patients showing a traininginduced improvement in alertness performance after training (3/4), there was reactivation of the right-hemisphere functional network known to subserve alertness in healthy participants. These reactivations were observed most robustly in right lateral PFC and medial frontal cortex but also in inferior parietal cortex. In contrast, a control group of healthy participants who also trained on the alertness paradigm showed decreases in the right frontoparietal alertness network. This was a different effect than that of the patients and was suggestive of traininginduced increases in neural efficiency, consistent with previous studies examining the effect of practice on higher cognitive functions, described in section 1. This latter finding again stresses the importance of taking into account participants' baseline cognitive ability and performance in the interpretation of practice-induced changes in functional activations.

In a similar study, Saur et $\mathrm{al}^{36}$ used fMRI to examine activations related to language processing in patients who suffered from aphasia caused by a left-hemisphere stroke. The patients were scanned 3 times: in the acute phase $(0-4 d$ poststroke), the subacute phase ( $\approx 2 \mathrm{wk}$ poststroke), and the chronic phase $(>4-12$ mo poststroke). The authors initially observed little activation of nonaffected language structures in the acute phase, followed by a large increase in right-hemisphere cognitive control and language areas in the subacute phase, which correlated with language improvement. Finally, in the chronic phase, the patients showed a normalization of activations, reflected in reduced right-hemisphere activations and reactivation of left-hemisphere language regions, in association with language recovery.

These findings suggest that restoration of function in injured brains can occur through reactivation of brain areas active in healthy brains during naive (pretraining) task performancethat is, by normalizing functional activations. There may also be cases where compensatory activations (recruitment of additional functional areas) are observed during the performance of cognitive tasks, as has been shown extensively in older adults. ${ }^{101-104}$ The use of neuroimaging and the examination of the effects of practice and training in such groups will help separate restorative and normalizing effects from compensatory effects. With further training, activations related to compensatory mechanisms may decrease, consistent with the normal pattern of redistribution of functional activations after practice. For example, Temple ${ }^{35}$ predicted that the compensatory activations they observed as a result of auditory and language training in dyslexic people would diminish with further training and improvements to performance. They also predicted that these changes would be accompanied by further normalization of activation in temporoparietal and frontal areas. Similarly, Saur, ${ }^{36}$ in an examination of language recovery in aphasic stroke patients, explained increased activations in right-hemisphere cognitive control areas in the subacute phase of language recovery in terms of increased demands on cognitive control processes while language areas are recovering but working at a suboptimal level. These demands are reduced in the later stage of recovery, in which continued language improvement is associated with a normalization of activations; reductions in right-hemisphere activations and increased engagement of left-hemisphere language-specific regions. These studies illustrate how compensatory effects can be dissociated from normalizing effects on the basis of neuroimaging examinations of practice effects and a knowledge of normal patterns of activation change, and thus, how a knowledge of normal patterns of brain plasticity is crucial to the interpretation of neurorehabilitative plasticity.

There are other potentially important applications of neuroimaging of brain plasticity in clinical populations. Although plastic changes in the brain often represent an adaptive response to injury and loss of input or function, there are many negative results of the brain's immense capacity for plasticity and reorganization. Phantom limb pain is an example of pathologic cortical reorganization in the absence of input, ${ }^{105,106}$ and focal hand dystonia in musicians is a result of repetitive movements associated with long-term and extensive practice. ${ }^{107,108}$ Neuroimaging interrogations of practice effects can also shed light on these processes. For example, Behrmann et $\mathrm{al}^{109}$ recently showed that training visual recognition of a set of artificial objects (Greebles) improved both Greeble recognition and common object recognition in a visual agnosic patient. However, these improvements were accompanied by increased impairment in face recognition. Functional imaging data enabled the authors to attribute this effect to a reorganization of function within the fusiform gyrus such that voxels that had previously been face-selective instead became Greeble-selective as a result of training. This study shows the potential use 
of neuroimaging technologies in assessing the effects of rehabilitative training efforts, particularly in localizing both the effects of injury and the effect of training, and in attempting to ensure that training does not have a negative impact on intact functions. As the prominent researcher Pascual-Leone has put it, our challenge is to modulate neural plasticity for optimal behavioral gain. ${ }^{110}$ Taken together, these recent studies show how this will be made possible: through the use of targeted cognitive training interventions that are grounded in a knowledge of patterns of normal brain plasticity to remediate cognitive and behavioral functioning and normalize brain activations.

\section{CONCLUSIONS}

The focus of this review has been the potential rehabilitation of cognitive functions. The literature on healthy control subjects can provide insights into experience-driven plasticity and guidance on methodologic considerations when interpreting activation changes. Our preliminary empirical data suggest that extensive practice on a verbal working memory task can lead to improvements in working memory-related processes and the ability to actively maintain information in working memory despite interference, improvement which may generalize to improved performance on other unpracticed working memory tasks. These data suggest that deficits in cognitive control may be ameliorated or rehabilitated through practice and training, a suggestion that merits further exploration in rehabilitative settings. Progress in our understanding of these effects will be enabled by neuroimaging examinations of cognitive rehabilitation training that are grounded in a knowledge of normal patterns of brain activation and practice-induced plasticity.

\section{References}

1. Holloway M. The mutable brain. Sci Am 2003;289:78-85.

2. Buonomano DV, Merzenich MM. Cortical plasticity: from synapses to maps. Annu Rev Neurosci 1998;21:149-86.

3. Karni A, Meyer G, Rey-Hipolito C, et al. The acquisition of skilled motor performance: fast and slow experience-driven changes in primary motor cortex. Proc Natl Acad Sci U S A 1998; $95: 861-8$

4. Kolb B, Whishaw IQ. Brain plasticity and behavior. Annu Rev Psychol 1998;49:43-64.

5. Chollet F, Weiller C. Imaging recovery of function following brain injury. Curr Opin Neurobiol 1994;4:226-30.

6. Robertson IH, Murre JM. Rehabilitation of brain damage: brain plasticity and principles of guided recovery. Psychol Bull 1999; 125:544-75.

7. Schallert T, Leasure JL, Kolb B. Experience-associated structural events, subependymal cellular proliferative activity, and functional recovery after injury to the central nervous system. J Cereb Blood Flow Metab 2000;20:1513-28.

8. Kelly AM, Garavan H. Human functional neuroimaging of brain changes associated with practice. Cereb Cortex 2005;15:1089102.

9. Poldrack RA. Imaging brain plasticity: conceptual and methodological issues.-a theoretical review. Neuroimage 2000;12:113.

10. Duncan J, Miller EK. Cognitive focus through adaptive neural coding in the primate prefrontal cortex. In: Stuss DT, Knight RT, editors. Principles of frontal lobe function. Oxford: Oxford Univ Pr; 2002. p 278-91.

11. Garavan H, Kelley D, Rosen A, Rao SM, Stein EA. Practicerelated functional activation changes in a working memory task. Microsc Res Tech 2000;51:54-63.
12. Jansma JM, Ramsey NF, Slagter HA, Kahn RS. Functional anatomical correlates of controlled and automatic processing. J Cogn Neurosci 2001;13:730-43.

13. Sayala S, Sala JB, Courtney SM. Increased neural efficiency with repeated performance of a working memory task is informationtype dependent. Cereb Cortex 2006;16:609-17.

14. Hempel A, Giesel FL, Caraballo NM, Amann M, Meyer H, Wustenberg $\mathrm{T}$, et al. Plasticity of cortical activation related to working memory during training. Am J Psychiatry 2004;161: 745-7.

15. Beauchamp MH, Dagher A, Aston JA, Doyon J. Dynamic functional changes associated with cognitive skill learning of an adapted version of the Tower of London task. Neuroimage 2003;20:1649-60.

16. Andreasen NC, O'Leary DS, Cizadlo T, et al. II. PET studies of memory: novel versus practiced free recall of word lists. Neuroimage 1995;2:296-305.

17. Andreasen NC, O'Leary DS, Arndt S, et al. I. PET studies of memory: novel and practiced free recall of complex narratives. Neuroimage 1995;2:284-95.

18. Haier RJ, Siegel BV Jr, MacLachlan A, Soderling E, Lottenberg S, Buchsbaum MS. Regional glucose metabolic changes after learning a complex visuospatial/motor task: a positron emission tomographic study. Brain Res 1992;570:134-43.

19. Elbert T, Pantev C, Wienbruch C, Rockstroh B, Taub E. Increased cortical representation of the fingers of the left hand in string players. Science 1995;270:305-7.

20. Hund-Georgiadis M, von Cramon DY. Motor-learning-related changes in piano players and non-musicians revealed by functional magnetic-resonance signals. Exp Brain Res 1999;125:41725 .

21. Munte TF, Altenmuller E, Jancke L. The musician's brain as a model of neuroplasticity. Nat Rev Neurosci 2002;3:473-8.

22. Karni A, Meyer G, Jezzard P, Adams MM, Turner R, Ungerleider LG. Functional MRI evidence for adult motor cortex plasticity during motor skill learning. Nature 1995;377:155-8.

23. Petersen SE, van Mier H, Fiez JA, Raichle ME. The effects of practice on the functional anatomy of task performance. Proc Natl Acad Sci U S A 1998;95:853-60.

24. Shadmehr R, Holcomb HH. Neural correlates of motor memory consolidation. Science 1997;277:821-5.

25. Frutiger SA, Strother SC, Anderson JR, Sidtis JJ, Arnold JB, Rottenberg DA. Multivariate predictive relationship between kinematic and functional activation patterns in a PET study of visuomotor learning. Neuroimage 2000;12:515-27.

26. Sakai K, Hikosaka O, Miyauchi S, Takino R, Sasaki Y, Putz B. Transition of brain activation from frontal to parietal areas in visuomotor sequence learning. J Neurosci 1998;18:1827-40.

27. Hikosaka O, Miyashita K, Miyachi S, Sakai K, Lu X. Differential roles of the frontal cortex, basal ganglia, and cerebellum in visuomotor sequence learning. Neurobiol Learn Mem 1998;70: 137-49.

28. Hikosaka O, Nakahara H, Rand MK, et al. Parallel neural networks for learning sequential procedures [published erratum in: Trends Neurosci 1999;22: following 568]. Trends Neurosci 1999;22:464-71.

29. Debaere F, Wenderoth N, Sunaert S, Van Hecke P, Swinnen SP. Changes in brain activation during the acquisition of a new bimanual coordination task. Neuropsychologia 2004;42:855-67.

30. Seidler RD, Purushotham A, Kim SG, Ugurbil K, Willingham D, Ashe J. Cerebellum activation associated with performance change but not motor learning. Science 2002;296:2043-6.

31. Jueptner M, Stephan KM, Frith CD, Brooks DJ, Frackowiak RS, Passingham RE. Anatomy of motor learning. I. Frontal cortex and attention to action. J Neurophysiol 1997;7:1313-24. 
32. Grafton ST, Hazeltine E, Ivry RB. Functional anatomy of sequence learning in normal humans. J Cogn Neurosci 1995;7:497510 .

33. Doyon J, Owen AM, Petrides M, Sziklas V, Evans AC. Functional anatomy of visuomotor skill learning in human subjects examined with positron emission tomography. Eur J Neurosci 1996;8:637-48

34. Sturm W, Longoni F, Weis S, et al. Functional reorganisation in patients with right hemisphere stroke after training of alertness: a longitudinal PET and fMRI study in eight cases. Neuropsychologia 2004;42:434-50.

35. Temple E, Deutsch GK, Poldrack RA, et al. Neural deficits in children with dyslexia ameliorated by behavioral remediation: evidence from functional MRI. Proc Natl Acad Sci U S A 2003;100:2860-5.

36. Saur D, Lange R, Baumgaertner A, et al. Dynamics of language reorganization after stroke. Brain 2006;129(Pt 6):1371-84.

37. Raichle ME, Fiez JA, Videen TO, et al. Practice-related changes in human brain functional anatomy during nonmotor learning. Cereb Cortex 1994;4:8-26.

38. Poldrack RA, Desmond JE, Glover GH, Gabrieli JD. The neural basis of visual skill learning: an fMRI study of mirror reading. Cereb Cortex 1998;8:1-10.

39. Poldrack RA, Gabrieli JD. Characterizing the neural mechanisms of skill learning and repetition priming: evidence from mirror reading. Brain 2001;124(Pt 1):67-82.

40. Fletcher P, Buchel C, Josephs O, Friston K, Dolan R. Learningrelated neuronal responses in prefrontal cortex studied with functional neuroimaging. Cereb Cortex 1999;9:168-78.

41. Petersson KM, Elfgren C, Ingvar M. Dynamic changes in the functional anatomy of the human brain during recall of abstract designs related to practice. Neuropsychologia 1999;37:567-87.

42. Pascual-Leone A, Torres F. Plasticity of the sensorimotor cortex representation of the reading finger in Braille readers. Brain 1993;116(Pt 1):39-52.

43. Pantev C, Oostenveld R, Engelien A, Ross B, Roberts LE, Hoke M. Increased auditory cortical representation in musicians. Nature 1998;392:811-4.

44. Haier RJ, Siegel BV, MacLachlan A, Soderling E. Regional glucose metabolic changes after learning a complex visuospatial/ motor task: a positron emission tomographic study. Brain Res 1992;570:134-43.

45. Landau SM, Schumacher EH, Garavan H, Druzgal TJ, D'Esposito M. A functional MRI study of the influence of practice on component processes of working memory. Neuroimage 2004;22:211-21

46. Asaad WF, Rainer G, Miller EK. Neural activity in the primate prefrontal cortex during associative learning. Neuron 1998;21: 1399-407.

47. Chen LL, Wise SP. Neuronal activity in the supplementary eye field during acquisition of conditional oculomotor associations. J Neurophysiol 1995;73:1101-21.

48. Chen LL, Wise SP. Supplementary eye field contrasted with the frontal eye field during acquisition of conditional oculomotor associations. J Neurophysiol 1995;73:1122-34.

49. Rainer G, Miller EK. Effects of visual experience on the representation of objects in the prefrontal cortex. Neuron 2000;27: $179-89$.

50. Buchel C, Coull JT, Friston KJ. The predictive value of changes in effective connectivity for human learning. Science 1999;283: 1538-41.

51. McIntosh AR, Rajah MN, Lobaugh NJ. Interactions of prefrontal cortex in relation to awareness in sensory learning. Science 1999;284:1531-3.
52. Ungerleider LG, Doyon J, Karni A. Imaging brain plasticity during motor skill learning. Neurobiol Learn Mem 2002;78:55364.

53. Korman M, Raz N, Flash T, Karni A. Multiple shifts in the representation of a motor sequence during the acquisition of skilled performance. Proc Natl Acad Sci U S A 2003;100: 12492-7.

54. Olesen PJ, Westerberg H, Klingberg T. Increased prefrontal and parietal activity after training of working memory. Nat Neurosci 2004;7:75-9.

55. Klingberg T, Forssberg H, Westerberg H. Training of working memory in children with ADHD. J Clin Exp Neuropsychol 2002;24:781-91.

56. Klingberg T, Fernell E, Olesen PJ, et al. Computerized training of working memory in children with ADHD-a randomized, controlled trial. J Am Acad Child Adolesc Psychiatry 2005;44: 177-86.

57. Cools R, Robbins TW. Chemistry of the adaptive mind. Philos Transact A Math Phys Eng Sci 2004;362:2871-88.

58. Egan MF, Goldberg TE, Kolachana BS, et al. Effect of COMT Val108/158 Met genotype on frontal lobe function and risk for schizophrenia. Proc Natl Acad Sci U S A 2001;98:6917-22.

59. Mattay VS, Goldberg TE, Fera F, et al. Catechol Omethyltransferase val158-met genotype and individual variation in the brain response to amphetamine. Proc Natl Acad Sci U S A 2003;100:6186-91.

60. Diamond A, Briand L, Fossella J, Gehlbach L. Genetic and neurochemical modulation of prefrontal cognitive functions in children. Am J Psychiatry 2004;161:125-32.

61. O'Connell MJ. Prediction of return to work following traumatic brain injury: intellectual, memory, and demographic variables. Rehabil Psychol 2000;45:212-7.

62. Grafman J, Salazar A, Weingartner H, Vance S, Amin D. The relationship of brain-tissue loss volume and lesion location to cognitive deficit. J Neurosci 1986;6:301-7.

63. Brooks N, McKinlay W, Symington C, Beattie A, Campsie L. Return to work within the first seven years of severe head injury. Brain Inj 1987;1:5-19.

64. Posner MI, Rothbart MK. Influencing brain networks: implications for education. Trends Cogn Sci 2005;9:99-103.

65. Rueda MR, Rothbart MK, McCandliss BD, Saccomanno L, Posner MI. Training, maturation, and genetic influences on the development of executive attention. Proc Natl Acad Sci U S A 2005;102:14931-6.

66. Sanes JN, Donoghue JP. Plasticity and primary motor cortex. Annu Rev Neurosci 2000;23:393-415.

67. van Mier H, Tempel LW, Perlmutter JS, Raichle ME, Petersen SE. Changes in brain activity during motor learning measured with PET: effects of hand of performance and practice. J Neurophysiol 1998;80:2177-99.

68. Kelly AM, Hester R, Foxe JJ, Shpaner M, Garavan H. Flexible cognitive control: effects of individual differences and brief practice on a complex cognitive task. Neuroimage 2006;31:86686

69. Raichle ME, MacLeod AM, Snyder AZ, Powers WJ, Gusnard DA, Shulman GL. A default mode of brain function. Proc Natl Acad Sci U S A 2001;98:676-82.

70. Greicius MD, Krasnow B, Reiss AL, Menon V. Functional connectivity in the resting brain: a network analysis of the default mode hypothesis. Proc Natl Acad Sci U S A 2003;100:253-8.

71. Greicius MD, Srivastava G, Reiss AL, Menon V. Default-mode network activity distinguishes Alzheimer's disease from healthy aging: evidence from functional MRI. Proc Natl Acad Sci U S A 2004;101:4637-42. 
72. Kennedy DP, Redcay E, Courchesne E. Failing to deactivate: resting functional abnormalities in autism. Proc Natl Acad Sci U S A 2006;103:8275-80.

73. Peterson BS, Skudlarski P, Anderson AW, et al. A functional magnetic resonance imaging study of tic suppression in Tourette syndrome. Arch Gen Psychiatry 1998;55:326-33.

74. Ziemann U, Paulus W, Rothenberger A. Decreased motor inhibition in Tourette's disorder: evidence from transcranial magnetic stimulation. Am J Psychiatry 1997;154:1277-84.

75. Enright SJ, Beech AR. Reduced cognitive inhibition in obsessive-compulsive disorder. Br J Clin Psychol 1993;32:67-74.

76. Rosenberg DR, Keshavan MS. Toward a neurodevelopmental model of obsessive-compulsive disorder. Biol Psychiatry 1998; 43:623-40.

77. Casey BJ, Castellanos FX, Giedd JN, et al. Implication of right frontostriatal circuitry in response inhibition and attentiondeficit/hyperactivity disorder. J Am Acad Child Adolesc Psychiatry 1997;36:374-83.

78. Pliszka SR, Liotti M, Woldorff MG. Inhibitory control in children with attention-deficit/hyperactivity disorder: event-related potentials identify the processing component and timing of an impaired right-frontal response-inhibition mechanism. Biol Psychiatry 2000;48:238-46.

79. Curtis CE, Calkins ME, Grove WM, Feil KJ, Iacono WG. Saccadic disinhibition in patients with acute and remitted schizophrenia and their first-degree biological relatives. Am J Psychiatry 2001;158:100-6.

80. Rubia K, Russell T, Bullmore ET, et al. An fMRI study of reduced left prefrontal activation in schizophrenia during normal inhibitory function. Schizophr Res 2001;52:47-55.

81. Roche RA, Dockree PM, Garavan H, Foxe JJ, Robertson IH, O'Mara SM. EEG alpha power changes reflect response inhibition deficits after traumatic brain injury (TBI) in humans. Neurosci Lett 2004;362:1-5.

82. Stuss DT, Gow CA. Frontal dysfunction after traumatic brain injury. Neuropsychiatry Neuropsychol Behav Neurol 1992;5: 272-82.

83. Craik FI, Anderson ND. Applying cognitive research to the problems of aging. In: Koriat A, Gopher D, editors. Attention and performance XVII. Cambridge: MIT Pr; 1999. p 583-615.

84. Verhaeghen P, Cerella J. Aging, executive control, and attention: a review of meta-analyses. Neurosci Biobehav Rev 2002;26:84957.

85. West RL. An application of prefrontal cortex function theory to cognitive aging. Psychol Bull 1996;120:272-92.

86. Cicerone KD, Dahlberg C, Kalmar K, et al. Evidence-based cognitive rehabilitation: recommendations for clinical practice. Arch Phys Med Rehabil 2000;81:1596-615.

87. Cicerone KD, Dahlberg C, Malec JF, et al. Evidence-based cognitive rehabilitation: updated review of the literature from 1998 through 2002. Arch Phys Med Rehabil 2005;86:1681-92.

88. Twamley EW, Jeste DV, Bellack AS. A review of cognitive training in schizophrenia. Schizophr Bull 2003;29:359-82.

89. Duncan J. Attention, intelligence and the frontal lobes. In: Gazzaniga MS, editor. The cognitive neurosciences. Cambridge: MIT Pr; 1995. p 721-33.

90. Duncan J, Owen AM. Common regions of the human frontal lobe recruited by diverse cognitive demands. Trends Neurosci 2000;23:475-83.
91. Kane MJ, Engle RW. The role of prefrontal cortex in workingmemory capacity, executive attention, and general fluid intelligence: an individual-differences perspective. Psychol Bull Rev 2002;9:637-71.

92. Engle RW, Kane MJ. Executive attention, working memory capacity, and a two-factor theory of cognitive control. In: Ross $\mathrm{B}$, editor. The psychology of learning and motivation. Vol 44 New York: Academic Pr; 2004. p 145-99.

93. Gray JR, Chabris CF, Braver TS. Neural mechanisms of general fluid intelligence. Nat Neurosci 2003;6:316-22.

94. Cicerone KD, Giacino JT. Remediation of executive function deficits after traumatic brain injury. NeuroRehabilitation 1992; 2:12-22.

95. Cicerone KD. Remediation of 'working attention' in mild traumatic brain injury. Brain Inj 2002;16:185-95.

96. Levine B, Robertson IH, Clare L, et al. Rehabilitation of executive functioning: an experimental-clinical validation of goal management training. J Int Neuropsychol Soc 2000;6: 299-312.

97. Wexler BE, Anderson M, Fulbright RK, Gore JC. Preliminary evidence of improved verbal working memory performance and normalization of task-related frontal lobe activation in schizophrenia following cognitive exercises. Am J Psychiatry 2000; 157:1694-7

98. Paulesu E, Demonet JF, Fazio F, et al. Dyslexia: cultural diversity and biological unity. Science 2001;291:2165-7.

99. Rumsey JM, Andreason P, Zametkin AJ, et al. Failure to activate the left temporoparietal cortex in dyslexia. An oxygen 15 positron emission tomographic study [published erratum in: Arch Neurol 1994;51:243]. Arch Neurol 1992;49:527-34.

100. Temple E. Brain mechanisms in normal and dyslexic readers. Curr Opin Neurobiol 2002;12:178-83.

101. Cabeza R. Hemispheric asymmetry reduction in older adults: the HAROLD model. Psychol Aging 2002;17:85-100.

102. Cabeza R, Anderson ND, Locantore JK, McIntosh AR. Aging gracefully: compensatory brain activity in high-performing older adults. Neuroimage 2002;17:1394-402.

103. Reuter-Lorenz PA, Jonides J, Smith EE, et al. Age differences in the frontal lateralization of verbal and spatial working memory revealed by PET. J Cogn Neurosci 2000;12:174-87.

104. Reuter-Lorenz P. New visions of the aging mind and brain Trends Cogn Sci 2002;6:394.

105. Ramachandran VS, Hirstein W. The perception of phantom limbs. The D. O. Hebb lecture. Brain 1998;121(Pt 9):1603-30.

106. Ramachandran VS, Rogers-Ramachandran D. Phantom limbs and neural plasticity. Arch Neurol 2000;57:317-20.

107. Elbert T, Candia V, Altenmuller E, et al. Alteration of digital representations in somatosensory cortex in focal hand dystonia. Neuroreport 1998;9:3571-5.

108. Pujol J, Roset-Llobet J, Rosines-Cubells D, et al. Brain cortical activation during guitar-induced hand dystonia studied by functional MRI. Neuroimage 2000;12:257-67.

109. Behrmann M, Marotta J, Gauthier I, Tarr MJ, McKeeff TJ. Behavioral change and its neural correlates in visual agnosia after expertise training. J Cogn Neurosci 2005;17:554-68.

110. Pascual-Leone A, Amedi A, Fregni F, Merabet LB. The plastic human brain cortex. Annu Rev Neurosci 2005;28:377-401. 